\title{
Stereoscopic motion analysis in densely packed clusters: 3D analysis of the shimmering behaviour in Giant honey bees
}

Gerald Kastberger ${ }^{1 *}$, Michael Maurer ${ }^{2}$, Frank Weihmann ${ }^{1}$, Matthias Ruether ${ }^{2}$, Thomas Hoetzl ${ }^{1}$, Ilse Kranner $^{3}$, Horst Bischof ${ }^{2}$

\begin{abstract}
Background: The detailed interpretation of mass phenomena such as human escape panic or swarm behaviour in birds, fish and insects requires detailed analysis of the 3D movements of individual participants. Here, we describe the adaptation of a 3D stereoscopic imaging method to measure the positional coordinates of individual agents in densely packed clusters. The method was applied to study behavioural aspects of shimmering in Giant honeybees, a collective defence behaviour that deters predatory wasps by visual cues, whereby individual bees flip their abdomen upwards in a split second, producing Mexican wave-like patterns.

Results: Stereoscopic imaging provided non-invasive, automated, simultaneous, in-situ 3D measurements of hundreds of bees on the nest surface regarding their thoracic position and orientation of the body length axis. Segmentation was the basis for the stereo matching, which defined correspondences of individual bees in pairs of stereo images. Stereo-matched "agent bees" were re-identified in subsequent frames by the tracking procedure and triangulated into real-world coordinates. These algorithms were required to calculate the three spatial motion components ( $d x$ : horizontal, $d y$ : vertical and dz: towards and from the comb) of individual bees over time.

Conclusions: The method enables the assessment of the 3D positions of individual Giant honeybees, which is not possible with single-view cameras. The method can be applied to distinguish at the individual bee level active movements of the thoraces produced by abdominal flipping from passive motions generated by the moving bee curtain. The data provide evidence that the z-deflections of thoraces are potential cues for colony-intrinsic communication. The method helps to understand the phenomenon of collective decision-making through mechanoceptive synchronization and to associate shimmering with the principles of wave propagation. With further, minor modifications, the method could be used to study aspects of other mass phenomena that involve active and passive movements of individual agents in densely packed clusters.
\end{abstract}

\section{Background}

Giant honeybee (Apis dorsata) nests [1-7] constitute a matrix of densely clustered individuals arranged in a multi-layered stratum, the "bee curtain" [8], around a central, flat comb (Figures 1 and 2). Collective behaviours such as mass flight activity and colony defence $[7,9]$ are affected by the functional principles of the 3D architecture of this bee curtain. Defence strategies against predatory wasps $[10,11]$ include shimmering

\footnotetext{
* Correspondence: gerald.kastberger@uni-graz.at

${ }^{1}$ Institute of Zoology, University Graz, Graz, Austria

Full list of author information is available at the end of the article
}

behaviour [3,12,13] (see Additional File 1, Movie S1), the ultimate evolutionary goals of which are not fully understood. Proximate aspects such as the underlying logistic principles of wave generation and propagation are also unclear [13]. Shimmering waves are produced by individual surface bees that consecutively flip their abdomens upwards, typically at an angle of $90^{\circ}[14]$ within $100 \mathrm{~ms}$. Information may be transmitted by "bucket-bridging" from one surface bee to an adjacent one, similar to Mexican waves in football stadiums [15], where information is also transferred by repetitive movements of individual participants consecutively. Shimmering waves can also "jump" from one excited

\section{Biomed Central}




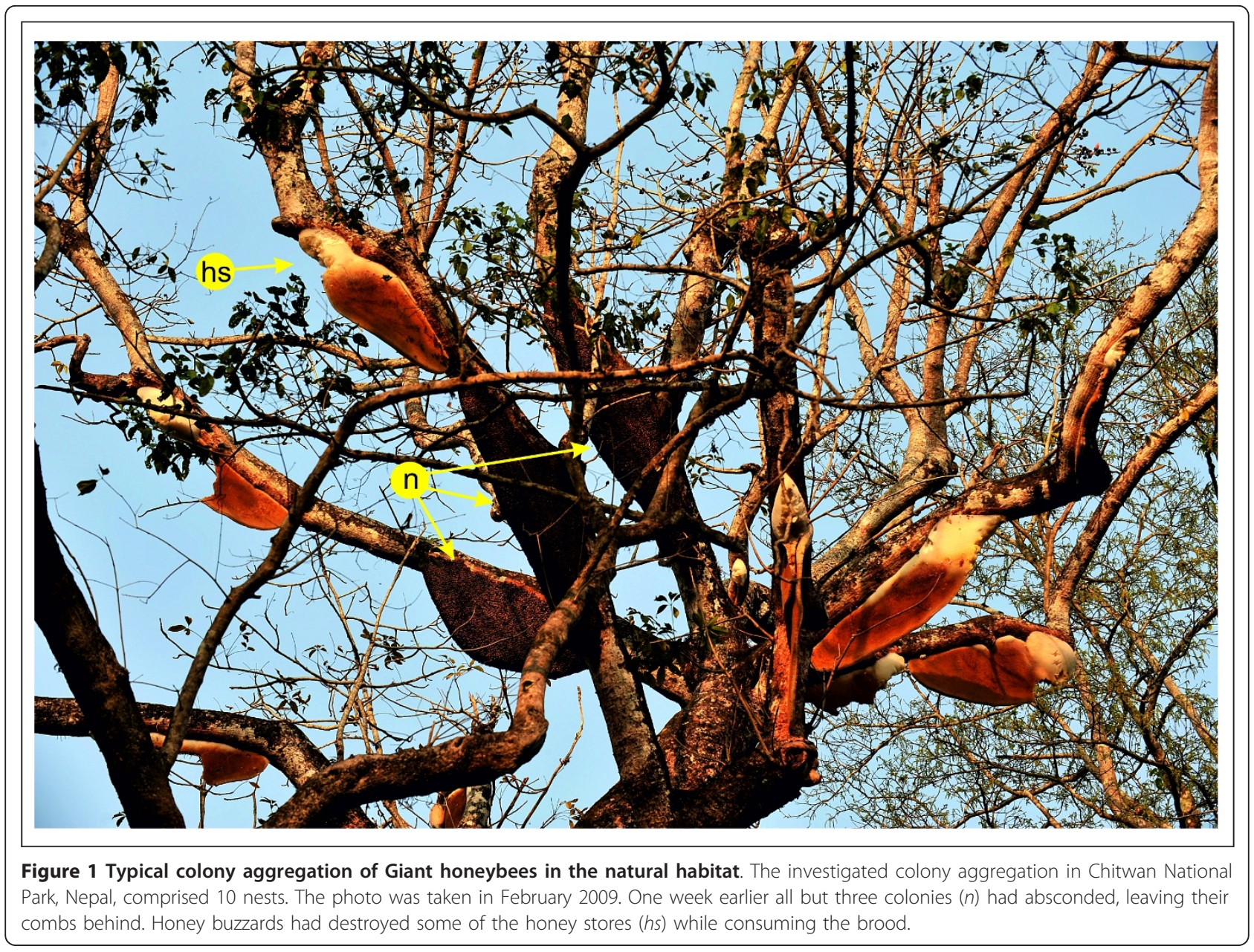

group of surface bees to another [13], which can cooccur with bucket-bridging. Ultimately, shimmering may not only provide visual patterns for external addressees such as predatory wasps, but may also play roles in colony-intrinsic communication $[13,16]$. We hypothesized $[13,16,17]$ that shimmering surface bees affect subsurface layers in the bee curtain, providing mechanoceptive signals for the colony members in all curtain layers, including those that do not actively participate in a wave. We studied shimmering in Giant honeybees under field conditions (Figure 1 and 2) in Chitwan (Nepal), with the goal to simultaneously measure the motions of hundreds of surface bees in the three directions of space to obtain a detailed view of the movements of individual bees within the entirety of the bee curtain. This was achieved by an adaptation of the stereoscopic imaging principle [18] with its fundamental algorithms (Figure 3): segmentation [19], matching [20,21] and reconstruction $[22,23]$ by tracking and triangulation.

Challenges arose, firstly, from the requirement to individually track identified surface bees, hereafter termed "agent bees", in successive frames (ff) of a shimmering process, which is difficult because all agent bees are extremely similar in morphology, are densely clustered, and show rapid movements in 3D during their abdominal flipping. Secondly, an individual bee sensing an incoming wave front due to the movements of her neighbours is free to decide whether or not to participate, and if so, whether to participate strongly or weakly. It is critical to distinguish active "movements", i.e. abdominal flipping, from passive "motions" caused by the surrounding bee curtain. Individuals that participate weakly in shimmering are difficult to detect by automated analysis. Thirdly, data of the positional coordinates of agents are, for the external observer, stochastic and noisy. This is because collective behaviours in eusocial insects are determined by self-organization [13,24-26], whereby patterns at a global level of a system emerge from numerous interactions among the lowerlevel components of the system, and rules specifying interactions among the systems' components are executed using only local information, without reference to the global pattern [24]. Hence, the data describing the behaviour of the lower-level components (here: the 

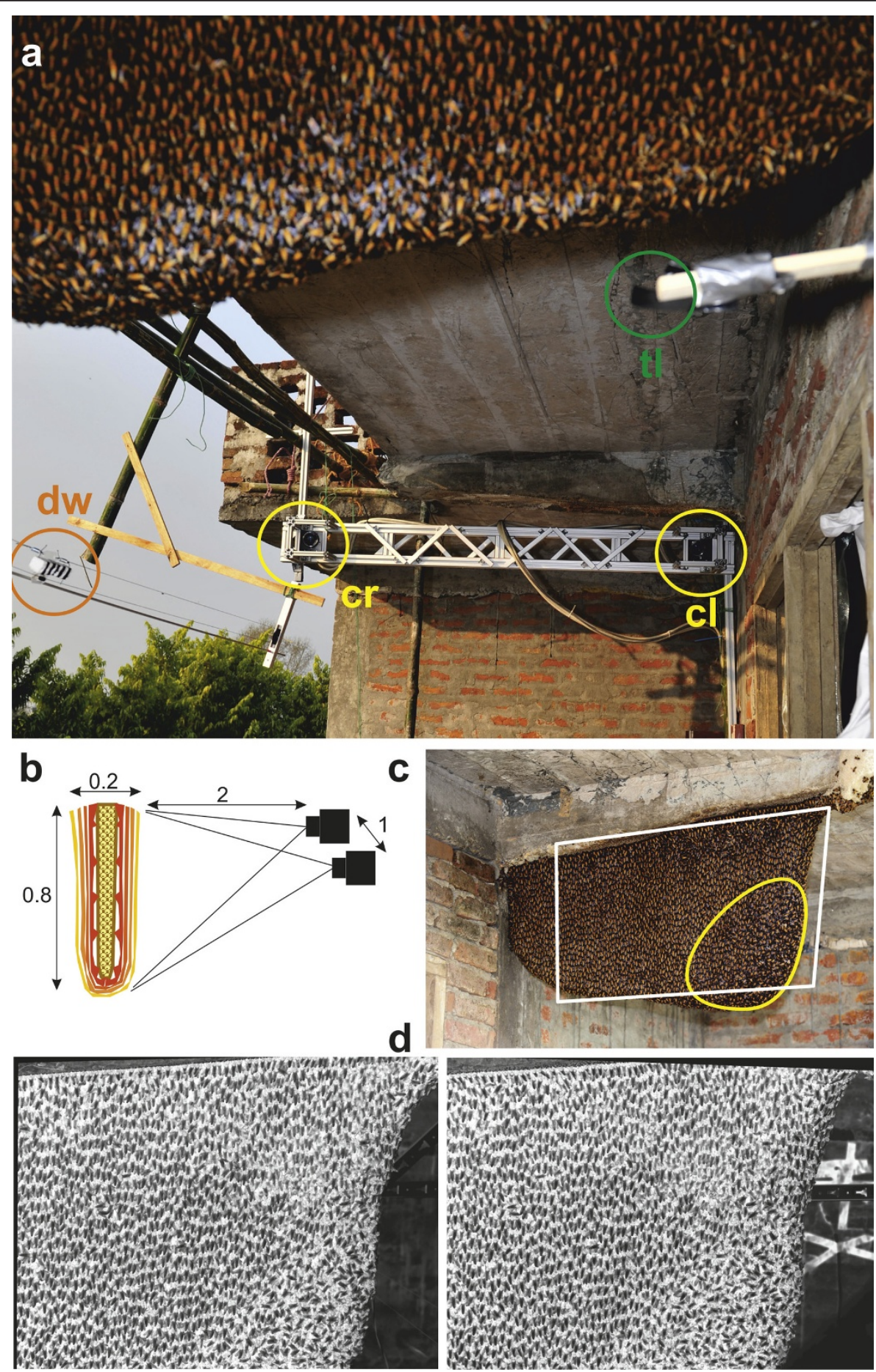

Figure 2 Acquisition of stereo images. (a) Pair of synchronized cameras ( $c_{r}$, right camera; $c_{1}$, left camera, yellow circles) with a resolution of $2352 \times 1728$ pixels at $60 \mathrm{~Hz}$ recording stereo images of a Giant honeybee nest on a balcony of a house in Chitwan, Nepal. Before the measurements, the cameras were fixed to an aluminium carrier rig and calibrated; the orange circle refers the movable dummy wasp (dw; note the black and white stripes) on a cable-car device; tl, trigger light (green circle) for the synchronization of the HD cameras and additional sensory equipment ( $\mathrm{cf}$ see Additional File 1, Movie S1). The frame grabbers and the computer were located in the adjacent room to the right side. (b) Schematic showing the camera setup at a Giant honeybee nest with a central comb with several layers of worker bees (termed "bee curtain"); numbers indicate distances in metres. (c) HD image of the experimental giant honeybee nest. The white rectangle indicates the area recorded by stereo imaging. The yellow line marks the "mouth zone", which is the most active zone of the nest where bees depart and arrive. (d) Example of a pair (left and right) of stereo images as acquired by the stereo cameras. 


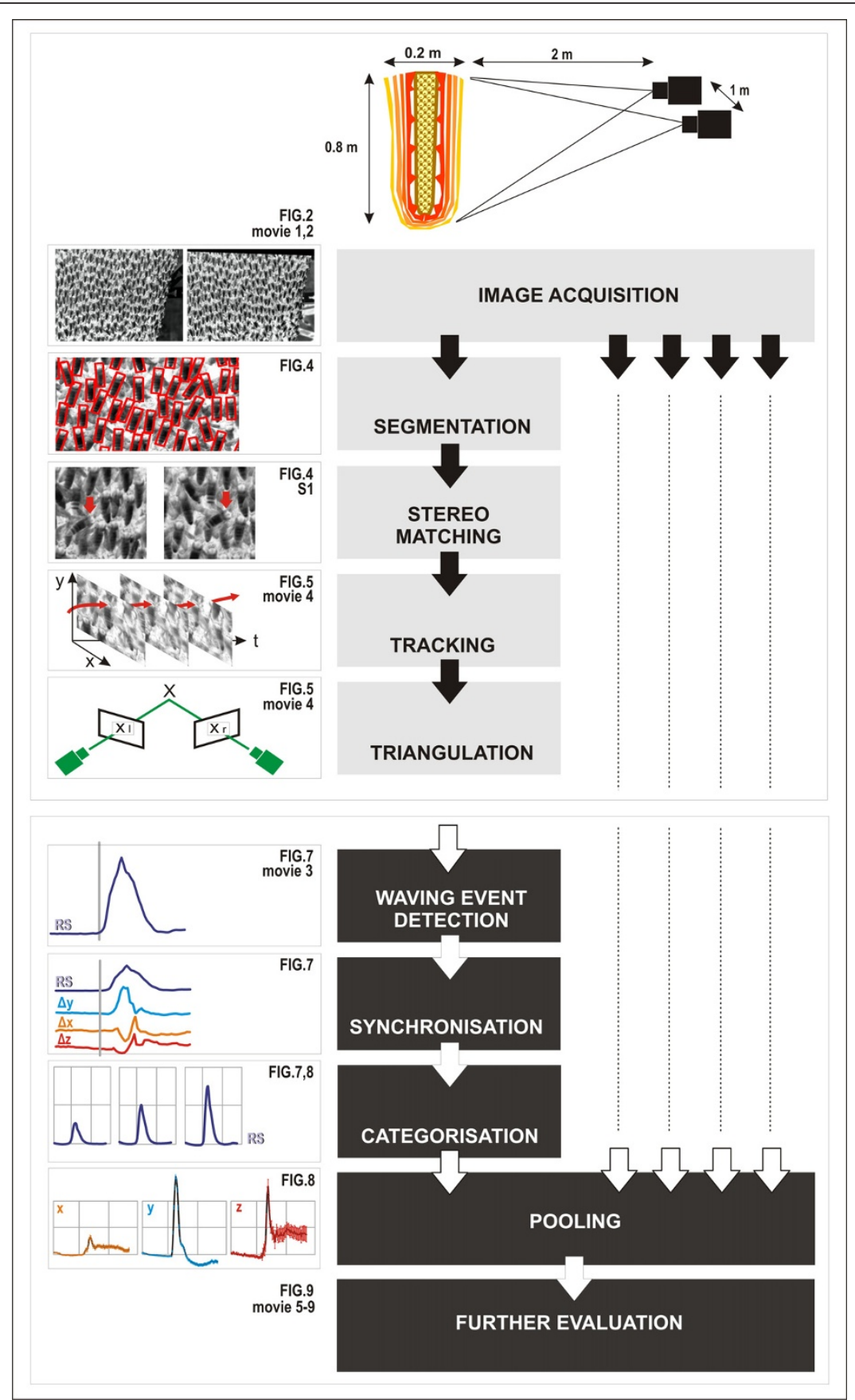

Figure 3 Schematic of the processes of 3D stereoscopic imaging and its application to the analysis of shimmering waves in Giant honeybees. From top to bottom: The experimental nest was captured by two frame-locked video cameras positioned at an angle of $30^{\circ}$ two metres in front of the nest. In the offline data assessment phase, the acquired images were processed as follows: Segmentation distinguished single agent bees in the densely packed clusters of bees on the surface of the nest; stereo matching identified corresponding agent bees in both paired images. These two processes enabled stereo tracking of the agents in subsequent frames throughout whole film scenes and triangulation of their thoracic positions regarding the three dimensions of space $(x, y, z)$. Following the stereoscopic process, the images are evaluated. For example, the arrival of the wave front at an individual agent can be recognized by a movement detection algorithm also producing a measure for the response strength RS of the agent. This allows synchronization and pooling of 3D data ( $\Delta \mathrm{x}, \Delta \mathrm{y}, \Delta \mathrm{z})$ of wave "episodes" (for definition, see text) of individual agent bees at different positions of the nest for a variety of behavioural applications. The bright-grey flow charts address the stereoscopic single-agent analysis from segmentation to categorization, the dark-grey flow charts refer to further processing such as synchronization and pooling of single-agent data. The arrows and dashed lines at the right symbolize that the stereoscopic process produced further data for hundreds of agent bees simultaneously. The panels to the left of the flow charts illustrate the results of the processes shown to their right (see referenced figures and movies for details). 
agent bees) appear stochastic and noisy, although the global effect (here: shimmering) is clearly recognisable. Fourthly, high-resolution and high-speed cameras are essential to produce rich data sets with high geometrical resolution, detecting movements of single agents within fractions of a millimetre across an entire nest that can span up to $1.5 \mathrm{~m}$ in diameter. The equipment must deliver reliable, accurate data, even under harsh field conditions in the natural habitats of Giant honeybees where electronic equipment may fail due to high temperature, air humidity and solar irradiance. Lastly, Giant honeybees are among the most aggressive insects known [8]. To avoid unwanted colony arousal, data recording must be non-invasive, keeping a distance of at least $2 \mathrm{~m}$ between equipment and nest.

We propose a method for the automated identification of individual bees within the densely packed clusters of the bee curtain surface, including the computation of the 3D locations of individual agent bees, following them over time and identifying the arrival of shimmering waves at those agents. The data obtained with this method can enhance our understanding of the generation and propagation of shimmering waves in Giant honeybees and of the contribution of nest members to intra-colonial communication.

\section{Results}

To automate the analysis of roughly 500 agents in a series of about 900 images per video sequence, we designed and implemented an algorithm using stereo videos as the input to calculate a three-dimensional movement path for each bee. In the following, we first describe in the Technical section (Figures 2 and 3) the implementation of image acquisition and of the chain of algorithms by which agent bees were identified and tracked. In each pair of stereo images, hundreds of surface bees were identified regarding their position and orientation in the images (Figure 4a-c). This segmentation was the basis for the stereo matching (Figure 4d-e and 5), which defines correspondences of individuals in pairs of stereo images. Stereo-matched agents were re-identified in subsequent frames by the tracking procedure and triangulated into real-world coordinates regarding their 3D locations and orientations (see Additional Files 2, 3 and 4, Movies S2, S3 and S4). In parallel, the arrival of shimmering waves at the selected agents was automatically detected within a path of stereo images. "Wave events" that triggered a response of an individual agent (to flip the abdomen or stay quiescent) were used as a means for synchronizing wave movement paths of hundreds of agents to achieve appropriate statistical evaluation of the data. In the Application section of this paper, we exemplified the use of the method to achieve a deeper understanding of selected behavioural aspects and to develop a model that describes the physical principles of abdominal flipping.

\section{Technical Section: Algorithm and Implementation Image acquisition}

At the experimental nest, the individual and collective motions of surface bees were captured in a stereoscopic video sequence (Figure 2). Pairs of synchronous, framelocked images recorded from different viewpoints ("stereo images") were obtained by two digital cameras (for technical specifications, see Additional File 5, Table S1) placed symmetrically $\sim 2 \mathrm{~m}$ in front of the nest with a baseline of $1 \mathrm{~m}$ between the cameras. The cameras were mounted on a solid carrier and connected to a computer. To elicit shimmering waves, a striped dummy wasp made from Styrofoam was fastened with fine yarn to a cable-car device for computer-controlled movements at variable velocity $(0.1-0.5 \mathrm{~m} / \mathrm{s})$ and direction (Figure 2). In the experiments described here, the dummy wasp was moved at an angle of $90^{\circ}$ to the nest surface (shown on the right side of the experimental nest in the images, see Additional File 1, Movie S1 and Additional File 6, Movie S5).

The stereo cameras had a resolution of $2352 \times 1728$ pixel $(p x)$. From the given working distance of $2 \mathrm{~m}$, and with a calibrated focal length of $53 \mathrm{~mm}$, about two thirds (700 $\mathrm{mm}$ in diameter) of the nest were recorded, whereby one $p x$ represented $\sim 0.30 \mathrm{~mm}$ in metric real-world coordinates. Therefore, a bee with an abdomen width of $6 \mathrm{~mm}$ was captured by an image region of roughly $20 p x$. The cameras were able to capture 60 frames per sec $(f p s)$ and so resolve the abdomen-flipping phase of an individual bee of $200 \mathrm{~ms}$ within 12 frames (ff). The resulting data stream of $480 \mathrm{MB} / \mathrm{s}$ was buffered to RAM and subsequently stored to disk. Consequently, the total of $8 \mathrm{~GB}$ of existing RAM allowed an acquisition time not longer than $15 \mathrm{~s}$. The cameras were connected as master and slave; one camera generated a trigger signal for the second camera to achieve temporal synchronization. The cameras were mounted on a carrier rig (Figure 2), which enabled their positioning at variable baselines $b$ (typically $1 \mathrm{~m}$ ) with a variable stereo angle $\alpha$. The baseline was calculated from the required working distance of $d=2 \mathrm{~m}$ and a stereo angle of $\alpha=30^{\circ}$ by

$$
b=2 d \tan \left(\frac{\alpha}{2}\right)
$$

The expected depth error $e_{z}$ was estimated on the assumption of orthogonal cameras according to

$$
e_{z}=\frac{1}{\sin \alpha} m e_{i m g} \cos \frac{\alpha}{2}
$$




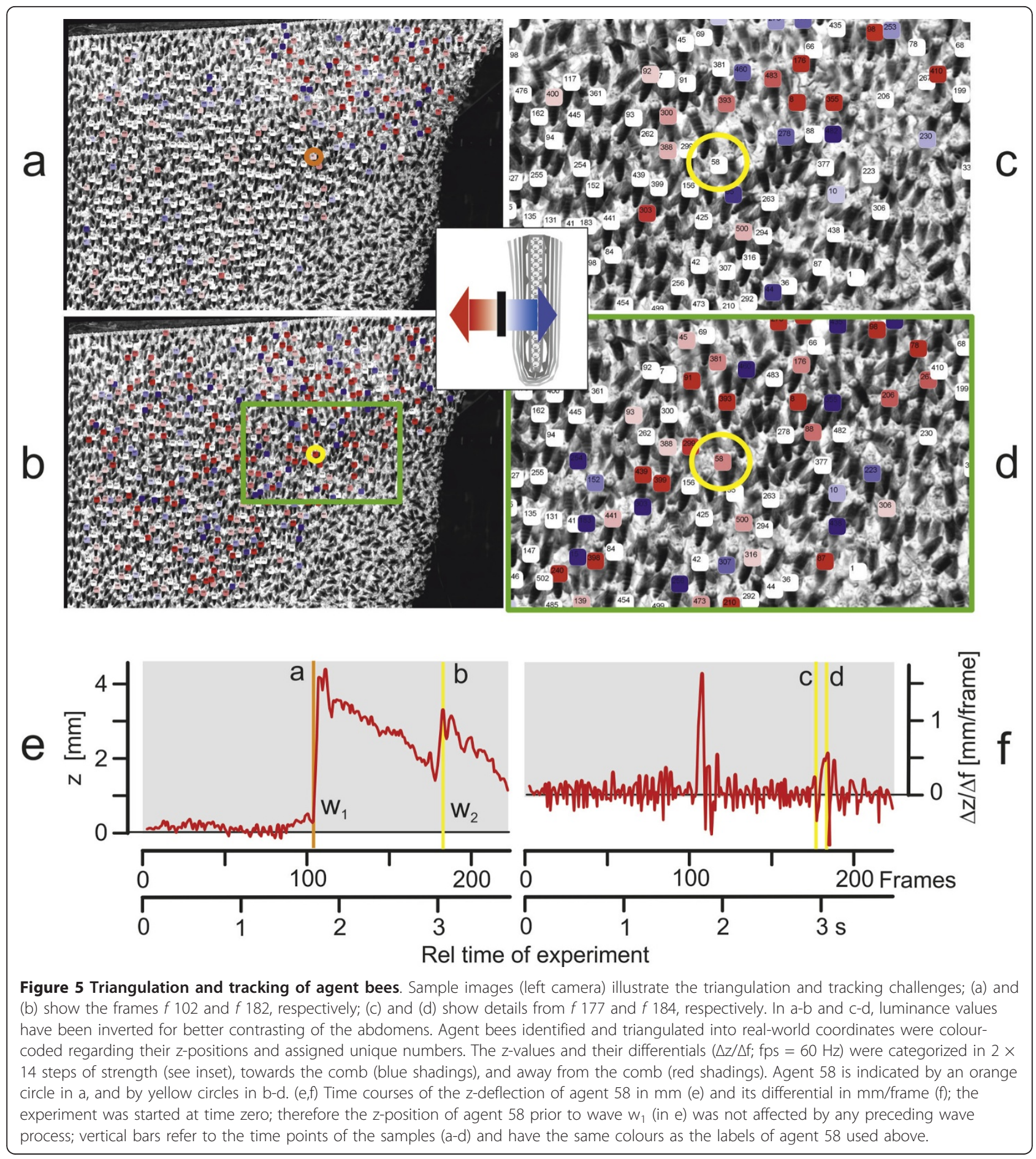

with $e_{i m g}$ as the pixel error of stereo matching; $m$, the magnification at the given working distance. Under the given conditions $\left(\alpha=30^{\circ}, m=0.3 \mathrm{~mm} / p x ; \mathrm{e}_{\mathrm{img}}=1 p x\right.$; equation 2) a resulting depth error of $e_{z}=0.6 \mathrm{~mm}$ was achieved. In summary, stereo images were typically recorded over $15 \mathrm{~s}$, capturing $900 \mathrm{ff}$ per camera at a rate of $60 \mathrm{~Hz}$ and at a spatial resolution of $0.3 \mathrm{~mm}$ per $p x$ within a measurement volume of $700 \times 500 \times 150 \mathrm{~mm}^{3}$ $(\mathrm{x}, \mathrm{y}, \mathrm{z})$ whereby the nest-specific axes were defined as $x$ (= horizontal: left-right), $y$ (= vertical: up-down) and $z$ (= directions towards and away from the comb).

\section{Segmentation}

Agents were segmented by comparison with pre-generated template images (Figure 4a). Normalized cross-correlation 
(NCC) [27] was used to define similarity. The correlation of an image $f_{m \times n}$ with a smaller two-dimensional template pattern $t_{k \times l}$, which shows a sample bee, was assessed according to

$$
c(u, v)=\sum_{\mathrm{x}, \mathrm{y}} f(x, y) t(x-u, y-v)
$$

To calculate the correlation for a single pixel location in $f$ with coordinates $(u, v)$, the template $t$ was overlaid to the region around this pixel and the sum of products of overlaying pixels within this region was calculated. In equation $3 x$ and $y$ denote the coordinates of all overlaying pixels in this region. Normalized cross correlation extends classical correlation and is robust to variations in illumination, and is formulated by normalizing the image and the template vectors to the unit length of the image according to

$$
\begin{aligned}
& \gamma 1(u, v)=\left[f(x, y)-\bar{f}_{u, v}\right] \\
& \gamma 2(u, v)=[\mathrm{t}(x-u, y-v)-\bar{t}] \\
& \gamma(u, v)=\frac{\sum_{\mathrm{x}, \mathrm{y}} \gamma 1(u, v) \ddagger 2(u, v)}{\sqrt{\sum_{x, y} \gamma 1(u, v)^{2} \sum_{x, y} \gamma 2(u, v)^{2}}}
\end{aligned}
$$

Here, $\bar{t}$ is the mean of $t$, and $\bar{f}_{u, v}$ is the mean of $f$ in the template.

The goal was to identify individual bees at the surface of a densely structured matrix in a multi-layered nest (Figures 1 and 2). For that, template patterns were defined from representative agent bees with varying orientations and scales (Figure 4a); such templates included the abdomen and the thorax rather than the head which is often concealed by other surface bees. Each template type was matched against every position $(u, v)$ in the image. Local similarity maxima, which were obtained by non-maximum suppression, represented successful matches. To compensate for overlaps, weak local maxima in the vicinity of $20 p x$ around a more dominant one were eliminated. Consecutively, the segmentation routine was repeated with scaled and rotated templates to account for variations between the individual prototypes in alignment and size. The charts (Figure 4b,c) exemplify template matching using five templates with a variation in orientation of $23^{\circ}$ (which represented a typical measure of the maximum deviation angle in the quiescent [7] areas of the nest), and with a scale tolerance of $14 \%$ (corresponding to $9 p x$ ). The procedure recognized hundreds of surface bees in the bee curtain (Figure 2c and 4c) under quiescent [7] conditions when they were inside the orientation limits and were not overlapped by other bees.

\section{Stereo matching}

Stereo matching allowed us to compare the template region of an agent in the left image with the paired, frame-synchronized right image, identifying the best match for the correct correspondence. This process could have followed the same strategy as that in segmentation. However, the high degree in pattern similarity of neighbours and the differences in perspective made it difficult to assign unique correspondences. Figure 4d,e illustrates the challenges arising in stereo matching exemplifying rectified honeybee templates: the agent segmented in the left stereo image coded by a yellow frame was identified in the paired right images by the similarity criterion and selected out of nine candidates; finally, the template matching was successful for the agent bee in the red rectangle.

From the segmentation algorithm, the thorax positions of $\mathrm{N}$ individuals in the left image and $\mathrm{M}$ individuals in the right image were known. This is given by

$$
\begin{aligned}
& S_{\text {left }}=\left\{t_{p}^{\text {left }}, p=1 . . N\right\} \\
& S_{\text {right }}=\left\{t_{i}^{\text {right }}, i=1 . . M\right\}
\end{aligned}
$$

To calculate the three-dimensional locations of segmented thoraces, we first identified a set of correspondences $c_{n}=(p, i)$, which followed the rule that $t_{p}^{\text {left }}$ and $t_{i}^{\text {right }}$ were images of the same thorax. One such correspondence is marked in Figure 4e. It is important to note that both stereo images were rectified [22], with the consequence that correspondences can only lie in the same row of both images. However, assessing the correspondence between agent bees was still challenging because of the presence of patterns with repetitive similarity. The method we used to solve this problem as a discrete energy minimization task is described in detail by

$$
\begin{aligned}
& \arg _{d} \min E(d) \\
& E(d)=E_{\text {data }}(d)+\lambda E_{\text {smooth }}(d)
\end{aligned}
$$

and in Additional File 7, Text S1 and Additional File 8, Figure S1 [28,29].

\section{Tracking}

The thoraces of agents were stereo tracked throughout the stereo sequence to obtain their 3D motions (Figure 5 and 6, see Additional Files 2, 3 and 4, Movies S2, S3 and $\mathrm{S} 4)$. The tracking method was closely related to the segmentation process; it defined a $20 \times 20 p x$ template 


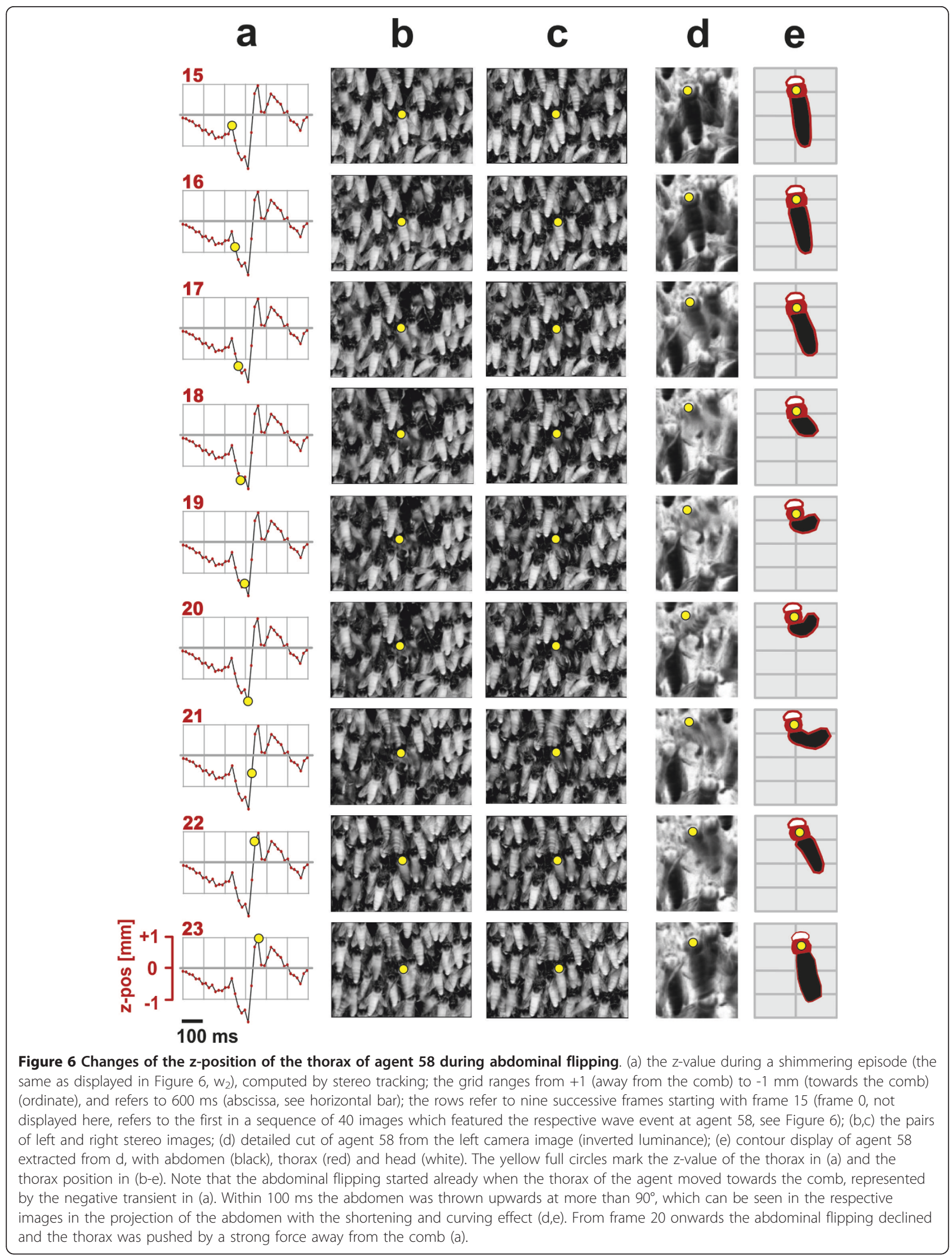


around the thorax of a segmented agent at time $t$ and compared it with a larger search region of $40 \times 40 p x$ in the subsequent image at time $t+1$. The location with the maximum NCC value was selected as an agent's new position. Here, the rapid, forceful abdominal thrusts of shimmering agent bees (Figure 6; see Additional Files 9, 10 and 11, Movies S6, S7 and S8) hampered the tracking success and required a higher robustness of the matching procedure. Utilizing a symmetry criterion deduced from the time course of the abdominal movements of each agent (Figure 6d-e), the search region in the current frame was compared with a set of $20 \times 20 p x$ templates from the preceding 15 frames. Consequently, the templates produced in the upward thrusting phase were successively compared with those from the downward phase. The tracking results obtained from the left and right images were finally fused by projecting them to their mean image row. This double matching produced accurate results avoiding drifts of the tracking points during abdominal flipping.

Despite the introduction of the symmetry criterion in the tracking procedure up to $16 \%$ of the $400-600$ identified agents were "lost" in the course of multiple waves. Nevertheless, we were able to compensate for these losses by restarting segmentation and stereo matching after each wave to retain stable numbers of agents throughout the evaluation path. We also minimized standard errors by the offset-correction of the positional coordinates of each agent. The arithmetic mean of the first six of the initial 30 frames prior to the arrival of a wave was subtracted from all data in a 90-frames episode (1500 ms). Offset correction also compensated for the residual spatial deflections with a time constant of 2-3 s which was characteristic for the descending process after a shimmering wave process caused by the receding motion of the bee curtain exemplified in Figure 5a-b and in the Additional File 3. Movie S3 shows the original data before offset-correction and prove that 3D stereoscopic imaging delivers the positional data without high-pass filter effects.

\section{Triangulation}

From each known correspondence $c_{n}=(p, i)$, the threedimensional thorax location $T_{n}$ was calculated by triangulation [22]. The known thorax locations in the image space and $t_{p}^{\text {left }}$ and $t_{i}^{\text {right }}$, in combination with known camera projection matrices $P^{l e f t}$ and $P^{\text {right }}$, were used to formulate the linear problem in

$$
\left[\begin{array}{cc}
{\left[t_{p}^{\text {left }}\right]_{\times}} & P^{\text {left }} \\
{\left[t_{i}^{\text {right }}\right]_{\times}} & P^{\text {right }}
\end{array}\right] T_{n}=0
$$

The term $[h]_{\times}$here denotes the $3 \times 3$ cross matrix of a three-dimensional vector $h$ (see [22]). A least-squares solution to the homogeneous equation system was obtained by solving the linear equation system. To maintain accuracy under the given field conditions, the stereo imaging system was calibrated daily using a standard calibration method [30] to maintain geometric consistency.

\section{Detecting the arrival of a wave at individual agents}

For the diagnosis of the behaviours of agent bees it is essential to distinguish active movements (caused by actively flipping the abdomen) from passive motions (caused by the motions of the bee curtain in the surrounding of an agent). For that, the precise time of the arrival of a wave at the agent's position must be determined. A reliable trigger criterion was found by detecting the luminance changes in two sequential frames $\left(f_{\mathrm{i}-1}\right.$, $f_{\mathrm{i}}$ ) in a pixel-wise subtraction creating a difference image (Figure 7 and 8d, see Additional File 12, Movie S9). We defined a sensor region of interest $\left(\mathrm{sROI}_{a}\right)$ of $60 \times 60 p x$, corresponding to $18 \times 18 \mathrm{~mm}$ in real-world coordinates, around each agent's thorax and recorded the mean difference in $\mathrm{sROI}_{a}$ luminance by $\Delta L_{a}=\Delta l u m\left(f_{\mathrm{i}-1}, f_{\mathrm{i}}\right)$, out of $3600 p x$. The size of $\mathrm{sROI}_{a}$ was chosen in conformance with the mean side-to-side distances between surface bees $(12.67 \pm 1.79 \mathrm{~mm}$; mean $\pm \mathrm{ME}, \mathrm{n}=50)$. $\Delta L_{a}$ was used to quantify the effect of an arriving wave at an individual agent; the noise level of $\Delta L_{a} \leq 5$ (corresponding to "black" in the difference image) represented the state "motionless" and $\Delta L_{a}=255$ ("white") "maximum movement". The arrival of a wave at an agent was defined two frames before the $\Delta L_{a}$ values had exceeded the threshold luminance value $\Delta L_{t h}\left(\Delta L_{a}>\Delta L_{t h}\right.$ with $\Delta L_{t h}=10$ ) within three successive frames (Figure 7 and $8 d$ ). Typically, $\Delta L_{a}$ peaked within $100 \mathrm{~ms}$, and the maximal values defined the "response strength" $(R S)$. For each agent, we considered thirty frames $(500 \mathrm{~ms})$ before and sixty frames $(1000 \mathrm{~ms})$ after time zero of the arrival of the wave and linked the $R S$ values with the positional 3D data. Detection of incoming waves was compromised if the distances between neighbouring bees were smaller than $10 \mathrm{~mm}$, whereby inactive agents could be erroneously classified as active in close vicinity with active neighbours. If required, correction is possible by reducing the area of $\mathrm{sROI}_{a}$. Here, we chose not to do so, because such compromising cases were rare and the errors were compensated for by the wealth of data recorded (Figure 8).

\section{Application Section: Behavioural Studies}

To demonstrate the applicability of the method we focused on selected behavioural aspects regarding shimmering waves. Here, data of only a single spreading direction were analysed for waves seemingly spreading from the right side to the left side of the experimental nest $\left(\mathrm{n}_{\text {waves }}=14 ; \mathrm{n}_{\text {episodes }}=4672 ; \mathrm{n}_{\text {agents }}=605 ;\right.$ when $\mathrm{a}$ shimmering wave hits an individual agent bee, a 


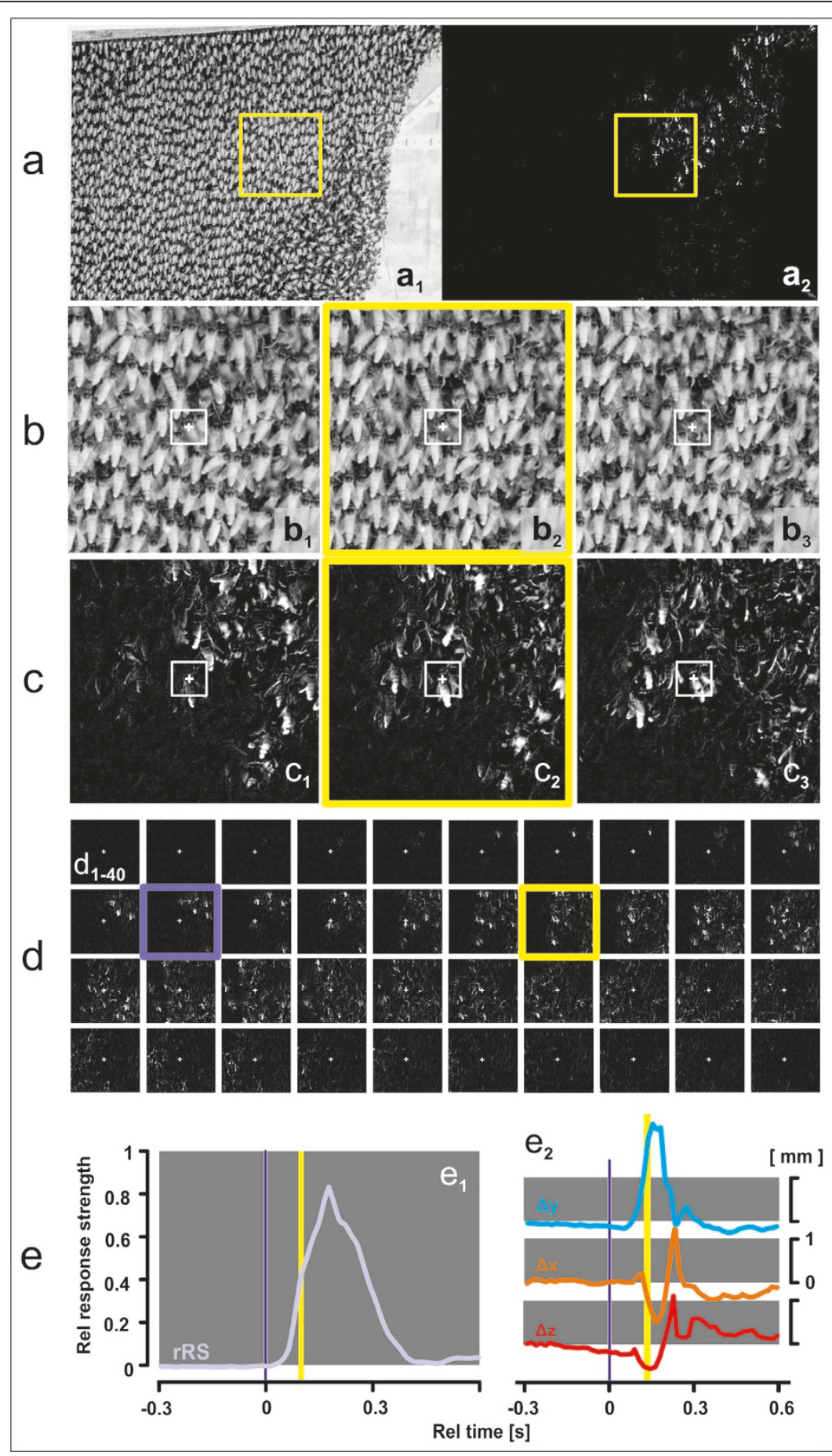

Figure 7 Detection of the wave front. (a) Frame ("f") 177 (of 900, taken by the left camera within $15 \mathrm{~s}$ at fps $=60 \mathrm{~Hz}$ ) recorded 6 frames after the arrival of a wave at agent 58 (compare vertical yellow lines in e); $\left(a_{1}\right)$ original image; $\left(a_{2}\right)$ differential image calculated by pixel-operated subtraction; the white spots show changes in luminance from $f 176$ to $f 177$. Yellow rectangles show $500 \times 500$ pixel areas around agent 58 for the frame 177. $\left(b_{1-3,}, c_{1-3}\right)$ Details of ff 176-178 (images from left to right) as original and differential images; white rectangles $(15 \times 15 \mathrm{~mm})$ denote the sensor regions of interest (sROls) for measuring the motions of (and around) agent 58 (labelled with a white cross). ( $\left.d_{1-40}\right)$ Survey of the differential images, exemplified in $\left(c_{1-3}\right)$, but for 40 frames; the arrival of the wave ( $t_{0}=0$; violet vertical bars in e) was calculated for $f 171$ (violet rectangles in d) and identified as the time at one frame before agent 58 was affected by the incoming wave. $\left(e_{1}\right)$ Relative response strength (rRS), expressed relative to the maximum RS value found in 605 agents. ( $\mathrm{e}_{2}$ ) Relative (offset-corrected, see text) changes of the positions of the thorax of agent 58 in the course of shimmering in $\mathrm{mm}$ : orange, horizontal directions ( $\Delta \mathrm{x}$ : positive direction is to the right side); blue, vertical directions ( $\Delta y$ : positive direction is upwards); red, directions towards and from the comb ( $\Delta z$ : positive direction is away from the comb). 


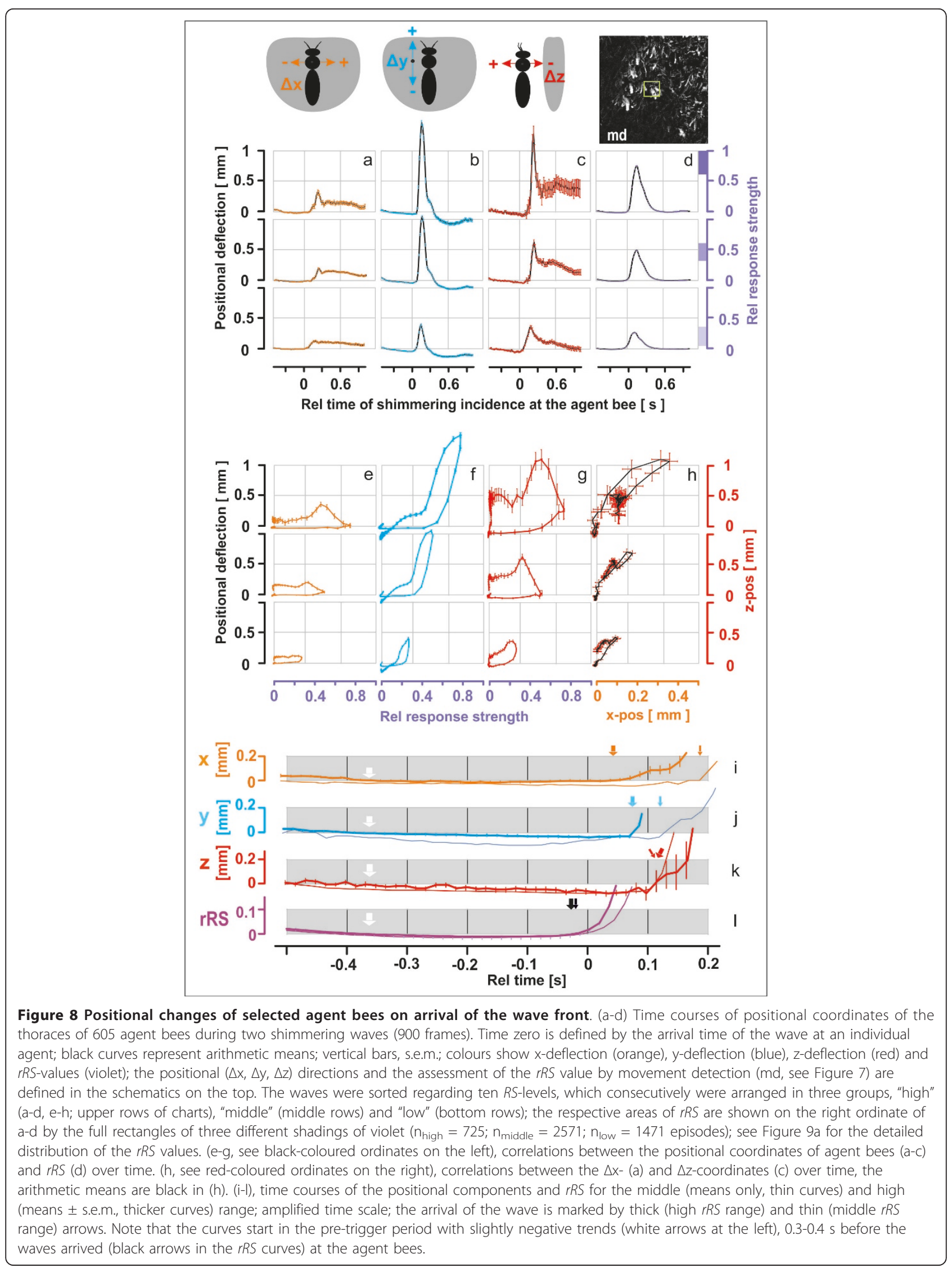


shimmering "episode" can be defined which includes the active and passive movements of this agent bee shortly before, during and after the arrival of the wave front). For all these behavioural examples, the statistical proof is based on synchronizing shimmering episodes by detecting the arrival of the wave at the immediate neighbourhood of the agent bees and on stereo tracking thoracic positions. In the first subchapter the primary motion components of selected agent bees are identified (Figure 5, 6, 7 and 8, see Additional File 2, Movie S2) which could be potential candidates to enforce the mechanical process of wave generation [31-33]. Second, for the same agents their (passively induced) motions were traced hundreds of milliseconds before the arrival of a wave, that is when the decision has to be made if and when to participate (Figure $8 \mathrm{i}-\mathrm{l}$ ) in a wave. Finally, we classified the modes of participation on the single agent level (Figure 6, 7 and 8d).

\section{Identifying primary motion components of agent bees during shimmering}

A shimmering wave arriving at an agent provokes characteristic positional changes (Figures 5, 6, 7 and 8, see Additional File 2, Movie S2). Regarding the y-components, $70 \mathrm{~ms}$ after wave arrival, as defined by the detection method described above (black arrows in Figure 81), a strong upward movement occurred (blue arrows in Figure $8 \mathrm{j}$ ); $20 \mathrm{~ms}$ later, the agent bee started to move in the $\mathrm{z}$-direction, first she shifted slightly negatively, i.e. towards the comb, then strongly away from the comb (red arrows in Figure 8k); this biphasic z-motion is displayed more explicitly in single-agent data (cf. Figures 6 and $\left.7 e_{2}\right)$. It is important to differentiate between the weak, positive transient deflections which resulted from the oncoming wave due to the general swinging of the bee curtain in the vicinity of the agent bee, and a strong, positive z-deflection which indicated "active" participation in the wave through abdominal flipping. With higher response strengths ( $R S$ values) the positive $\mathrm{y}$-deflections started earlier (Figure $8 \mathrm{j}$ ) and the positive $\mathrm{z}$-motions later (Figure $8 \mathrm{k}$ ) due to a more pronounced preceding negative phase of the $\mathrm{z}$-component (cf. Figure 6). The horizontal components were the weakest of all, and the data illustrate that the positive $\mathrm{x}$-components corresponded strongly with the positive $\mathrm{z}$-components $(\mathrm{r}$ $=0.39 ; \mathrm{P}<0.001 ; \mathrm{n}=4782$ wave episodes at agent bees; Pearson Product Moment Correlation Test); this happened at lower $R S$ values at a lower intensity and with increasing time lags (Figure $8 \mathrm{~h}, \mathrm{i}, \mathrm{k}$ ). The deflections regarding $\mathrm{x}-, \mathrm{y}$ - and $\mathrm{z}$-directions seem to be very complex because they are caused by the specific attributes of the functional architecture of the bee curtain regarding an agents orientation (head up and abdomen downwards) and the coupling (physical contact) between the colony members of the different layers
$[8,13,16,17]$. More details are given in the discussion part.

\section{Motion detection at an agent bee prior to the arrival of a wave}

To produce the typical cascadic shimmering waves that are visually recognized by external addressees [16], each surface bee needs to respond to an oncoming wave within a time window of less than $100 \mathrm{~ms}$. For that, she has to decide whether or not to flip the abdomen, and if she does, she can raise her abdomen with gradual strength. Her decision is particularly linked to mechanical cues sensed tens of milliseconds prior to the arrival of the wave front. The possibility that movements of her immediate neighbours are also perceived visually cannot be excluded, but it would probably take too long to synchronize her abdominal flipping with the wave front only by visual input; the duration of senso-neural processes associated with image perception may last more than $150 \mathrm{~ms}$ [34] to prompt behavioural decisions $[13,16,35]$. However, the visual domain is important, in particular to perceive threatening stimuli such as predatory wasps hovering around the nest. Such visual patterns are key to initialize shimmering $[13,16]$ and to collectively drive its spreading direction [35]. Mechanical cues, although less understood [13,16,35], must be essential for the wave spreading process. Surface bees are well endowed to sense mechanical cues, as they cling on to bees of the subsurface layer with their six legs, acting as a potent web of mechanoreceptors. In this paper, 3D stereoscopic imaging provided evidence for mechanoceptive cues, which were traced at individual agents in terms of small increments up to $300 \mathrm{~ms}$ before the wave front arrived (Figure 5e and 8i-k). Statistically proven for the investigated scenario of waves with high $R S$ values (see Figure 7a for the $\mathrm{z}$-component, test not shown here) spreading from the right to the left side, a wave typically pressed the agent bees slightly $(<0.2 \mathrm{~mm})$ towards the nest, then shifted them into the direction of the oncoming wave, and lastly slightly downwards. However, recordings of agent 58 (Figure 5 and 6) illustrate in two successive episodes that the sensing of incoming waves could be even more complex (see Figure 5e, episode $\mathrm{w}_{1}$ ).

\section{Participation of individual agent bees in shimmering}

Wave episodes were classified into ten response strength $(R S)$ levels (Figure 9) regarding the agents' participation in shimmering, but in Figure 8 they were summarized in only three groups, "low", "medium" and" high". The occurrence of shimmering strengths followed a Gaussian distribution $(\mathrm{P}<0.05$, Kolmogorow-Smirnow test; Figure 9a), which means that the majority of agents participated at medium $R S$. The variation in participation strength can be discerned in difference images (see Additional File 4, Movie S4) by their resulting 


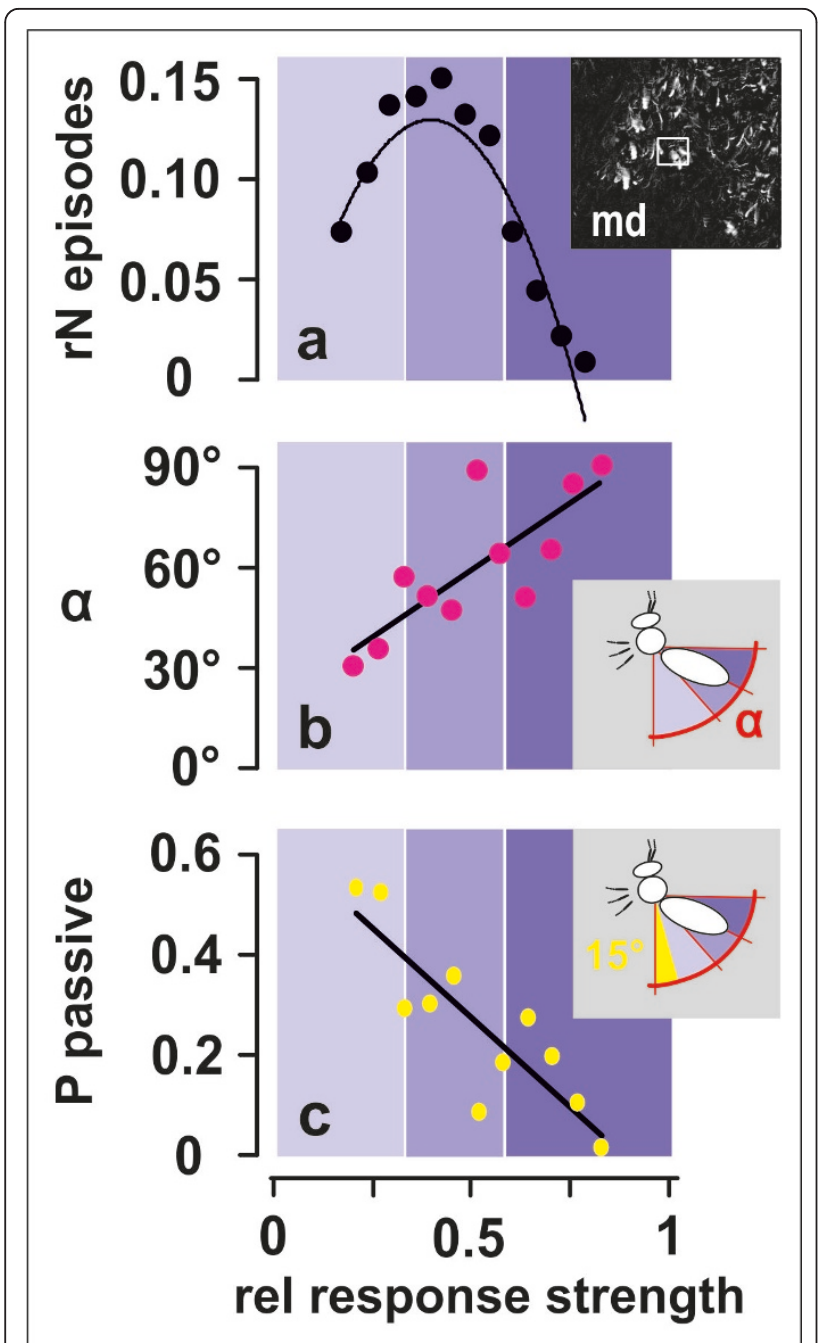

Figure 9 Correlation between response strength $R S$ and flipping angle $\boldsymbol{\alpha}$. The response strengths (RS) of agent bees assessed by movement detection (see inset: md; Figures 7c-e and 8) were normalized to the maximum value in 605 agents and sorted regarding 10 levels between 0.3 to 0.8 of the relative maximal strength (rRS). (a) Gauss distribution of the sorted episodes according to the rRS levels ( $r N$ episodes, relative number of episodes; $n_{\text {episodes }}=4672$ ). Three $r R S$ divisions were defined, marked by different shadings of the violet background. (b) Weighted means of flipping angles in a selected group of episodes (black regression line: $y=78.997 x+2.601 ; n_{\text {episodes_sel }}=176 ; R^{2}=0.626 ; P<0.01$, Spearman Rank Correlation test). (c) The probability of inactivity (no or weak abdominal flipping with flipping angles less than $15^{\circ}$ as detected by manual inspection) correlated negatively with the rRS (black regression line: $y=0.717 x+0.389 ; n_{\text {episodes_sel }}=176 ; R^{2}=$ 0.739; $\mathrm{P}<0.001$, Spearman Rank Correlation).

luminance $(\max \Delta l u m)$ values. On wave arrival, the motion components $(\mathrm{x}, \mathrm{y}, \mathrm{z}$ : Figure $8 \mathrm{a}-\mathrm{c})$ showed initial positive peaks which correlated with $R S$. At the lowest $R S$ level $(R S=1$; not displayed in Figure 8) these transient peaks were not visible, which means that strong positive deflections at higher $R S$ levels indicate "active" participation in shimmering. We cross-checked these results with the occurrence of abdominal flipping. In the stereo images (Figure 6d) the abdomens were projected as curved ellipses of decreasing lengths, which allowed assessing the momentary flipping angle. The $R S$ values correlated with these angles positively (Figure 9b), and negatively with the occurrence of "passiveness" as defined for Figure 9c whereas flipping angles of $0^{\circ}-15^{\circ}$ represented "passive" contribution. The data confirm that agent-specific $R S$ values, and therefore also their correlates, the transient positive positional deflections (Figure 8a-c), are usable measures for the participation in shimmering.

\section{Applications: Physical Principles of Abdominal Flipping}

We also explored the physics of abdominal flipping at the single bee level (Figure 6 and 7, see Additional Files 9, 10 and 11, Movies S6, S7 and S8) and the potential accompanying mechanical cues in colony-intrinsic communication [13]. The thoracic z-motions during shimmering exhibit a prominent initial peak (Figure 8c) due to the abdominal thrusting. This is exemplified for agent 58 , where we manually linked the thoracic position and the angular movement of abdominal flipping (Figure 6). Initially $\left(f f_{\text {rel }}=1-6\right.$, defined as $f f 15-20$ in Figure $\left.6 a\right)$, the thorax moved progressively towards the comb by $1 \mathrm{~mm}$ in $100 \mathrm{~ms}$, but then $\left(f f_{\mathrm{rel}}=7-9\right)$ it was strongly pushed away from the comb (see Additional File 2, Movie S2 and Additional Files 9, 10 and 11, Movies S6, S7 and S8) with a six-fold greater velocity.

Giant honeybees in the peripheral and outer layers of the bee curtain deposit a lot of watery honey in their abdomens [9], which become much heavier than the counterbalancing heads. Therefore, an abdomen-flipping bee may be simplified, in terms of classic mechanics, as a torsion pendulum (see Additional File 11, Movie S8) with an a-centric axis (positioned between thorax and the tarsi of the six legs which fix the thorax to the movable subsurface) and with two disparate masses (the upwards head and the downwards abdomen) on both sides of the rotation centre, oscillating in a (harmonic but damped) curved motion. During abdominal flipping mass inertia produces a reaction force, which initially presses the agent with the thorax towards the nest, but consecutively, the thrust of the flail-type body with the asymmetry of the pendular masses initiates a centrifugal force directed away from the comb. In the collective process of shimmering the centrifugal forces of the surface bees are locally synchronized, which lastly pulls the subsurface layers of the bee curtain concertedly away from the comb (see Additional Files 9, 10 and 11, Movies S6, S7 and S8). Thus, the centrifugal z-component in the second phase of abdominal flipping mainly drives the shimmering process. 


\section{Discussion}

Limits to image acquisition within the given stereoscopic approach

The above application was mainly limited by geometrical and technical constraints. Studying active movements and passive motions at a Giant honeybee nest, such as in shimmering behaviour, needs a thorough 3D analysis with the accuracy of a fraction of a millimetre. We aimed to measure the positional deflections at a resolution of $0.1 \mathrm{~mm}$ in all directions of space of hundreds of agent bees simultaneously. This required a stereo baseline of 1 $\mathrm{m}$, which made the stereo system rather large and sometimes difficult to handle under challenging field conditions. To maintain adequate calibration, the cameras were mounted on an aluminium girder that fixed the position of the cameras to each other and enabled movements of the two cameras in a fixed position in front of the bee colony if required. However, we had to compromise the temporal resolution with the illumination conditions at the scene. A nest may be positioned in a dark corner of a veranda or in the bright sun on a tree. Longer exposure times will produce motion blur during fast movements as occur during shimmering. Varying illumination at the nest site was compensated for by locally applied algorithms such as normalized similarity measures. We avoided excessive sunlight, which would prevent reliable measurement by overexposing of nest regions. To satisfy our needs in terms of accuracy and measurement time, two frame-synchronized cameras (see specifications in Additional File 5, Table S1) with a resolution of $4 M p x$ were used, with fixed lenses (without motorized zoom, focus or iris) to provide calibration stability, and with CMOS image sensors that provide flexible capturing of images of different size at different frame rates (ranging from 60 to more than $500 \mathrm{fps}$ ). Lastly, one of the main problems was the enormous data rate produced by the cameras during the experiments. At a frame rate of 60 images with $4 M p x$ recorded by each camera, an uncompressed data flow of about $480 \mathrm{MB} / \mathrm{s}$ had to be managed. Due to the limitation of electric power in the field during our expedition, it was not possible to use hard disks to capture the images in parallel. Therefore, the data had to be stored in the RAM of the computer and written to hard disks later on, constraining acquisition time to RAM storage capacity (in our case: 8 GB RAM, $15 \mathrm{~s}$ acquisition time).

\section{Simultaneous recording of hundreds of agents by stereoscopic imaging}

Existing optical tracking methods [36,37] mostly record single views (e.g. in CCTV cameras) of isolated agents in low quantities; if large quantities of agents are viewed, such as hundreds or thousands, motion priors are usually derived from the composite movement of "crowds" [30,37]. These single-view methods are inappropriate for mass phenomena such as shimmering in Giant honeybees, where the precise positional 3D coordinates of individual agents need to be known independently of the global motion. We chose to use 3D stereoscopic imaging, which allows motion analysis of densely packed agents in all directions of space. For this, we developed a system with stabilized tracking performance and resolved ambiguities, which identifies the 3D movements of hundreds of agents simultaneously. So far, shimmering has been documented on traditional film $[5,38]$ in conjunction with classical image analysis, providing only 2D projections of individual bees participating in a $3 \mathrm{D}$ process. Laser Doppler vibrometry (LDV) [39] can be useful for some applications, as it also facilitates $3 \mathrm{D}$ information. However, it has the disadvantage of delivering data of only one single agent bee over time, and can only pick up transient changes as it is limited by high-pass filter effects.

Here, the stereo tracking method has been modified to measure local, comparably small movements of hundreds of densely packed agents simultaneously, in a flat, but nevertheless three-dimensional stratum. The method is applicable not only to insect clusters such as Giant honeybee nests, but also to processes where positional changes over time have to be monitored on the surface of a stratum of agents with high packing density (such as the growth of plants or cells in $3 \mathrm{D}$, or for displaying deformations of any type of textured surface). For the application of stereoscopic imaging to monitor the dynamics of flocks of birds or fish schools, the methodological approach, in particular the tracking rules, must be adapted.

\section{Perspectives of analysing shimmering stereoscopically}

For the shimmering behaviour of Giant honeybees, the described stereoscopic method produced data that provide behavioural details that could not have been measured by other techniques. It provided evidence for a series of potential mechanoceptive cues perceived by hundreds of surface bees on arrival of a shimmering wave. For example, at the beginning of the experiment, agent 58 drifted slightly away from the comb $(<0.3$ $\mathrm{mm}$ ), starting $300 \mathrm{~ms}$ before it strongly participated in the shimmering wave (Figure 5e: episode $\mathrm{w}_{1}$, see the positive deflection at ff 102-115). These "pre-trigger" z-motions of the agents are apparently caused by the abdomen-flipping neighbours. Although small, such passive motions may enable the surface bees to estimate strength and spreading direction of an oncoming wave, and may function to trigger their active participation in shimmering. This response to the wave front is complex: first, the bodies were lifted upwards and pressed towards the comb, and also shifted towards the spreading wave 
front. Thereafter, the actively shimmering, abdomenlifting agents pull the subsurface layers massively away from the comb. We propose that the resulting biphasic time course of the z-motion is a subtle colony-intrinsic signal $[13,16,35]$ that provides mechanoceptive information about the momentary defensive state of the colony regarding temporal, spatial and directional patterns. The addressees of such signals are those colony members that are positioned in the subsurface layers and inactive in shimmering, and that are excluded from receiving visual cues.

Hence, 3D analysis of singular aspects of shimmering, such as the thorax positions of surface bees, enhances the understanding of the mechanical basis of abdominal flipping at the single bee level, and of the sensory basis for colony-intrinsic information involved in the spreading of shimmering waves. On the individual bee level, the stereoscopic method enabled a comparison of the mechanics of abdominal flipping with that of a torsion pendulum. On the colony level, it allowed an assessment of the mechanic basics of shimmering to explore potential cues for colony-intrinsic communication. In our example, the wave arrived from the right side and drew the agents against the spreading direction. The $\mathrm{x}$-motions of the agents detected can be plausibly explained by the mechanical principle of shear forces tangential to the individual agents. This is analogous to wind waves that propagate along the interface between water and air [31-33]; as the wind blows, pressure and friction forces perturb the equilibrium of the water surface, producing waves in which the moving paths of particles near the water surface form circles (regarding monochromatic, linear, and plane waves in deep water [31-33]). Wind waves are therefore a combination of longitudinal (back and forth) and transverse (up and down) motions $[23,32,33]$. The positive $\mathrm{x}$-motions of agents in horizontally spreading shimmering waves are supposed to correspond to the longitudinal moving of water particles $[23,32,33]$ when directed "backwards".

However, the analogy of shimmering waves with wind waves is limited for at least two reasons. First, wind waves are generated [33] by energy transferred onto the water whereas shimmering is produced by the "active" agents in the bee curtain themselves. Second, in wind waves the restoring force is gravity [33], but in shimmering waves it is the concerted muscular activity of the bees in the layers of the bee curtain acting rectangular to gravity. The positive z-motion in shimmering denotes that abdominal flipping has recruited energy into the previously quiescent parts of the bee curtain, pulling its elastic mass away from the comb with successive damped oscillations (Figure 5e; see Additional File 2, Movie S2 and Additional Files 9, 10 and 11, Movies S6, S7 and S8). This finding questions the analogy to
Mexican waves [15] in football stadiums: Although both shimmering and Mexican waves are generated by the energy of the participating agents, the audience in football stadiums does not lift the ground into the air, whereas the wave front during the shimmering process pushes the surface of the bee curtain to the outside direction.

\section{Conclusions}

So far, stereo imaging has been applied to remote sensing [40], close-range photogrammetry [41], material sciences [42,43], medicine $[44,45]$ and tissue mechanics [46]. Although this method can be scaled to a multitude of measurement ranges and corresponding accuracy specifications, to date 3D stereoscopic imaging has not been used for ethological purposes, particularly not for the descriptions of 3D motions of densely packed agents. We propose that it is a valuable tool to study collective behaviours in Giant honeybee colonies, but also, subject to additional adaptations of the method, for swarm behaviours in other insects $[47,48]$, fish $[49,50]$ and birds [51-54] and for special aspects of escape panic in humans [55].

\section{Additional material}

Additional file 1: Shimmering behaviour in Giant honeybees. An

experimental Giant honeybee nest $(140 \times 70 \mathrm{~cm})$ attached to a balcony of a hotel in Chitwan, Nepal. On the right, parts of the comb had been removed by a honey hunter some weeks before. With the exception of the mouth zone [8] (bottom right), the bee curtain exhibited a quiescent structure of surface bees, with their heads up and the abdomens down. The movie was recorded with a HD camera with a frame rate of $50 \mathrm{~Hz}$ in parallel to the stereo cameras (see Figure 2 for experimental design). At the beginning of the sequence the trigger light (fixed by black adhesive tapes to the wall behind) was turned on for one second. The red spot (middle of the right side of the nest) was produced by the beam of a Laser vibrometer measuring the thoracic z-position of the selected surface bee. Right to the nest, a black-and-white striped dummy wasp was mounted on the cable-car device, and its moving speed and direction were computer-controlled. The dummy wasp provoked shimmering waves before it is seen in the image. Note that the waves originated at the right nest side above the mouth zone. The yellow number on the right bottom gives the time in seconds. The first two waves of this sequence refer to the episodes $w_{1}, w_{2}$ of Figure 6 (2.1 MB, $M P G)$.

Additional file 2: Illustration of the triangulation of a selected agent by stereo tracking. The upper row shows image sequences of the left and right camera regarding the wave episodes $w_{1}$ and $w_{2}$ of agent 58 (for information about cut size and location, see Figure 6 and 7; for further details about z-movement and abdomen flipping, see Figure 7 and 9); the red crosses in the images mark the thorax of agent 58. The bottom bar chart gives the $z$-values of the thoracic position of agent 58 in $\mathrm{mm}$; positive values refer to directions away from the comb, negative directions towards the comb. The moving red line in the graph marks the momentary time position. Note that the waves in the episodes $w_{1}$ and $w_{2}$ spread from right to left, and that the participation in shimmering of the neighbours of agent 58 varied strongly (1.9 MB, MPG).

Additional file 3: Visualization of the z-movements of all selected agents by stereo tracking during a single wave episode. Part of the experimental Giant honeybee nest (cf. Additional File 1, Movie S1, Additional File 6, Movie S5 and Additional File 12, Movie S9). The left 
camera image displays the wave episode $w_{2}$ as shown in Additional File 2, Movie S2 (cf. Figure 6, 7 and 8). Matched and triangulated agents were marked with rounded rectangles showing the identification number. The colours used for coding the $z$-position refer to 14 steps of towards-comb direction (blue shades from white to dark blue) and offcomb direction (red shades from white to dark red). This film shows the positional information for all 505 agents (cf. Figure 6ab). Note that the waves cause residual motions of the curtain away from the comb for two seconds (as displayed in Figure 6e for agent 58). Numbers refer to frame and time (in ms) information (0.1 MB, MPG).

Additional file 4: Visualization of the differential of z-movements of all selected agents by stereo tracking during a single wave episode This film shows the differential of the positional information for all 505 agents (cf. Figure $6 c, d, f)$. For all other details, see legend to Additional File 3, Movie S3 (0.2 MB, MPG).

Additional file 5: Specification of the stereo cameras. The stereo imaging setup consisted of two global-shutter CMOS cameras, delivering $4 \mathrm{Mpx}$ gray-scale images at a frame rate of $60 \mathrm{~Hz}$. The images were recorded and stored by a battery-powered industrial PC.

Additional file 6: Stereo imaging of shimmering. Detail of the experimental Giant honeybee nest (cf. to Additional File 1, Movie S1). For comparison, the image sequence refers to the same $15 \mathrm{~s}$ displaying the shimmering waves shown in Additional File 1, Movie S1. Left and right images of the black-and-white high-speed stereo cameras with frame and time information; images are displayed in inverted luminance values. Note that due to the perspective only the left camera shows the cable car dummy (2.5 MB, MPG).

Additional file 7: Stereo matching: the correspondence problem described as a discrete energy minimization task. Detailed

description of the algorithm for stereo matching which allows automated identification of corresponding individuals in a pair of stereo images. The problem is challenging because of the inherent similarity of the colony members addressed as agent bees. The problem was formulated as a discrete energy minimization task.

Additional file 8: Stereo matching by identifying the minimum cut through a reduced graph. Each segmented bee in the left image was assigned a chain of $M$ disparity slots in the right image, according to equation 9. In this example, $M=3$, one chain corresponds to a column in the graph, and the number of bees in the left image corresponds to the number of columns. Each node contains at the most one bee in the right image, which lies in the respective disparity interval (equation 10). In this example, the red nodes contain a bee, whereas the white nodes are empty. Start and end of each chain are connected from a source to a sink node, respectively. Links in the graph (black and blue lines) are pairwise connections between nodes, and are assigned capacity values $C_{0}(p), C t(p, i)$ and $C n(p, q, i)$, according to equations 11,13 and 14 . Cutting of a t-link is equivalent to selecting the bee above the cut as the correct correspondence. The cut that completely separates source from sink (a special case is illustrated by the dashed red line) has the smallest sum of cut link capacities; it is called minimum cut [28], and results in an optimal assignment of correspondences.

Additional file 9: Illustration of abdominal flipping. Illustration of abdominal flipping of bees in the neighbourhood of agent 58 (marked by a red full circle) during the wave episode $w_{2}$ (see Figures 6,7 and 9). Footage in frames and time in ms are displayed (frame rate: $60 \mathrm{~Hz}$ ). For all other details, see legend to Additional File 3, Movie S3 (0.5 MB, MPG).

Additional file 10: Slow motion of abdominal flipping. Slow motion of abdominal flipping of bees in the neighbourhood of agent 58 (marked by a red full circle) during the wave episode $w_{2}$ (see Figure 6,7 and 9). Footage in frames and time in $\mathrm{ms}$ are displayed (factor slow. motion: 10.6). For all other details, see legend to Additional File 12, Movie S9 (2.2 MB, MPG).

Additional file 11: Mechanistic model of an abdomen-flipping Giant honeybee at the surface of a nest. Model, explaining the movements of the body associated with abdominal flipping according to the $y$ - and $z$-curves in Figure 9 (not considering the x-movements). The left brown vertical bar denotes the two-sided comb with cells on both sides separated by a mid wall. The two short brown vertical bars between the model bee and the model comb represent the subsurface layers of the bee curtain to which the model bee clings with her six extremities (not shown). In this model, the position of head and thorax, and the distance between thorax and the nearest brown bar was kept constant throughout the flipping process. In the film, the abdominal flipping is simplified: the model bee raises the abdomen by $90^{\circ}$, which provokes $y$ movements (here displayed in vertical directions) and z-movements (here displayed in horizontal directions). Two phases of the flipping are illustrated. In the initial phase the model bee is pressed towards the subsurface layers, when she actively pulls her body upwards using her extremities, and in the second phase, she moves away from the comb and recovers her initial lower position. In this sketch, the changing interspaces between comb and the subsurface layers additionally illustrate the pressing to and moving away from the comb of the model bee. Numbers indicate frames and time in $\mathrm{ms}$; the sketch slows down real-time abdominal flipping by a factor of 10.78 (0.5 MB, MPG).

Additional file 12: Difference image sequences. Detail of the experimental Giant honeybee nest (cf. Additional File 1, Movie S1 and Additional File 6, Movie S5). For comparison, the image sequence refers to the same $15 \mathrm{~s}$ displaying the shimmering waves shown in Additional File 6, Movie S5. Left, left-camera image; right, difference image giving the subtraction value of $\Delta l u m_{i,-1}=\mid \operatorname{lum}_{\mathrm{i}}-\operatorname{lum}_{\mathrm{i}-1}$ between the actual frame $f_{\mathrm{i}}$ and the preceding frame $f_{\mathrm{i}-1}$; numbers refer to frame and time (in ms) information; the left image shows inverted luminance values (2.5 $M B, M P G)$

\section{Acknowledgements}

We thank Dr. Madhusudan Man Singh from the Tribhuvan University, Kirtipur, Kathmandu, Nepal and three anonymous referees for comments that helped improving the manuscript. Financial grant was provided by the Austrian Science Fund (P 20515-B16).

\section{Author details}

'Institute of Zoology, University Graz, Graz, Austria. ${ }^{2}$ Institute for Computer Graphics and Vision, Graz University of Technology, Graz, Austria. ${ }^{3}$ Royal Botanic Gardens Kew, Wakehurst Place, UK.

\section{Authors' contributions}

Conceived and designed the experiments: GK. Performed the experiments: GK TH MM FW. Analyzed the data: GK TH MM FW. Contributed analysis tools: MM GK MR. Wrote the paper: GK MM MR IK. Co-editing: HB. All authors read and approved the final manuscript.

\section{Competing interests}

The authors declare that they have no competing interests.

Received: 23 October 2010 Accepted: 8 February 2011

Published: 8 February 2011

\section{References}

1. Roepke W: Beobachtungen an indischen Honigbienen, insbesondere an Apis dorsata. Meded LandbHoogesch Wageningen 1930, 34:1-28.

2. Lindauer M: Über die Verständigung bei indischen Bienen. Z Vergl Physiol 1956, 38:521-557.

3. Seeley TD, Seeley RH, Aratanakul P: Colony defence strategies of the honeybees in Thailand. Ecological monographs 1982, 52:43-63.

4. Ruttner F: Biogeography and taxonomy of honeybees Berlin: Springer Verlag; 1988.

5. Kastberger G: The Magic Trees of Assam - Documentary film about the biology of the Giant honeybee Apis dorsata. National Geographic, ZDF, ORF epo-film Wien 1999.

6. Randall Hepburn H, Hepburn C: Bibliography of the Giant honeybees, Apis dorsata Fabricius (1793) and Apis laboriosa F. Smith (1871). Apidologie 2007, 38:219-220.

7. Kastberger G, Winder O, Hoetzl T, Raspotnig G: Behavioural features of a periodic form of massed flight activity in the Giant honeybee Apis dorsata. Apidologie 1996, 27:381-395. 
8. Morse RA, Laigo FM: Apis dorsata in the Philippines. Philipp Assoc Ent 1969, 1:1-96.

9. Oldroyd BP, Wongsiri S: Asian honey bees: Conservation, and Human Interactions Cambridge: Harvard University Press; 2006.

10. Koeniger N, Fuchs S: Zur Kolonieverteidigung der asiatischen Honigbienen. Tierpsychologie 1975, 37:99-106.

11. Ono M, Igarashi T, Ohno E, Sasaki M: Unusual thermal defense by a honeybee against mass attack by hornets. Nature 1995, 377:334-336.

12. Kastberger G, Raspotnig G, Biswas S, Winder O: Evidence of Nasonov scenting in colony defence of the Giant honeybee Apis dorsata. Ethology 1998, 104:27-37.

13. Schmelzer $E$, Kastberger $G$ : "Special agents" trigger social waves in giant honeybees (Apis dorsata). Naturwissenschaften 2009, 96:1431-1441.

14. Woyke J, Wilde J, Wilde M: Comparison of Defense Body Movements of Apis laboriosa, Apis dorsata dorsata and Apis dorsata breviligula Honey Bees. J Insect Behav 2008, 21:481-494.

15. Farkas I, Helbig D, Vicsek T: Mexican waves in an excitable medium. Nature 2002, 419:131-132.

16. Kastberger G, Schmelzer E, Kranner I: Social waves in Giant honeybees repel hornets. PLOS ONE 2008, 3(9):e3141.

17. Kastberger G, Weihmann F, and Hoetzl T: Self-Assembly Processes in Honeybees: The Phenomenon of Shimmering. In Honeybees of Asia Edited by: Hepburn R, Radcliff S 2011, Chapter 18.

18. Wheatstone C: Contributions to the physiology of Vision - part the first. On some remarkable, and hitherto unobserved, phenomena of binocular vision. Philosophical Transactions 1838, 128:371-394.

19. Freixenet J, Muñoz X, Raba D, Martí J, Cufí X: Yet Another Survey on Image Segmentation: Region and Boundary Information Integration. Proceedings of the 7th European Conference on Computer Vision-Part III, LNCS 2002, 2352:408-422.

20. Scharstein D, Szeliski R: A taxonomy and evaluation of dense two-frame stereo correspondence algorithms. International Journal of Computer Vision 2002, 47:7-42.

21. Zureiki A, Devy M, Chatila R: Stereo Matching using Reduced-Graph Cuts. Proceedings Int. Conf. on Image Processing 2007, 1:237-240.

22. Hartley R, Zisserman A: Multiple View Geometry in Computer Vision Cambridge: Cambridge University Press; 2003.

23. Faugeras O: Three-Dimensional Computer Vision Cambridge: The MIT Press; 1993.

24. Camazine S, Deneubourg JL, Franks NR, Sneyd J, Theraula G, Bonabeau E: Self-organization in biological systems New Jersey: Princeton University Press; 2003.

25. Camazine S, Sneyd J: A model of collective nectar source selection by honey bees: self-organization through simple rules. J Theor Biol 1991, 149:547-571.

26. Pasteels JM, Deneubourg JL, Goss S: Self-organization mechanisms in ant societies [I]: trail recruitment to newly discovered food sources. In From individual to Collective Behavior in Social Insects. Edited by: Pasteels JM, Deneubourg JL. Basel: Birkhauser Verlag; 1987:155-175.

27. Lewis JP: Fast normalized cross-correlation. Vision Interface, Conf. Proceedings 1995, 1:120-123.

28. Goldberg AV, Tarjan RE: A New Approach to the Maximum Flow Problem. Journal of the ACM 1988, 35:921-940.

29. Gusfield D, Irving RW: The Stable Marriage Problem: Structure and Algorithms Cambridge: The MIT Press; 1989.

30. Zhang Z: A Flexible New Technique for Camera Calibration. IEEE Transactions on Pattern Analysis and Machine Intelligence 2000, 22(11):1330-1334.

31. Phillips OM: The dynamics of the upper ocean Cambridge University Press, USA; 1977.

32. Lamb H: Hydrodynamics Cambridge: Cambridge University Press; 1994.

33. Holthuijsen LH: Waves in oceanic and coastal waters Cambridge: Cambridge University Press; 2007.

34. VanRullen R, Thorpe SJ: The time course of visual processing: From early perception to decision-making. Journal of Cognitive Neuroscience 2001, 13(4):454-461.

35. Kastberger G, Weihmann F, Hoetzl T: Complex social waves of Giant honeybees provoked by a dummy wasp support the special-agent hypothesis. Communicative \& Integrative Biology 2010, 3(2):1-2.

36. Yilmaz A, Javed O, Shah M: Object Tracking: A Survey. ACM Comput Surv 2006, 38(4):Article 13.
37. Ali S, Shah M: Floor Fields for Tracking in High Density Crowd Scenes. Proceedings of 10th European Conference on Computer Vision, Computer Science 2008, 5303(2):1-14.

38. Kastberger $G$, Winder $O$, Steindl K: Defence strategies in the Giant honeybee Apis dorsata. Proceedings of the Deutsche Zoologische Gesellschaft: 4-8 June 2001; Osnabrück 2001, 94:1-7.

39. Weihmann F, Hoetzl T, Kastberger G: 3-D patterning of social waves in the Giant honeybee (Apis dorsata) [abstract]. DZG 102. Annual Meeting 2009, S26.

40. Kraus K: Photogrammetry. Geometry from images and laser scans Berlin/New York: de Gruyter; 2007.

41. Atkinson KB: Close Range Photogrammetry and Machine Vision Dunbeath: Whittles Publishing; 2001

42. Stampfl J, Scherer S, Gruber M, Kolednik O: Reconstruction of surface topographies by scanning electron microscopy for application in fracture research. Applied Physics A 1996, 63:341-346.

43. Pouchou JL, Boivin D, Beauchêne P, Vignon F: 3D Reconstruction of Rough Surfaces by SEM Stereo Imaging. Microchimica Acta 2002, 139:135-144.

44. Stoyanov D, Darzi A, Yang GZ: Dense 3D depth recovery for soft tissue deformation during robotically assisted laparoscopic surgery. International Conference on Medical Image Computing and Computer Assisted Intervention 2004, 2:41-48.

45. Lau WW, Ramey NA, Corso J, Thakor NV, Hager GD: Stereo-Based Endoscopic Tracking of Cardiac Surface Deformation. Internationa Conference on Medical Image Computing and Computer Assisted Intervention 2004, 1:494-501.

46. Pierce DM, Trobin W, Trattnig S, Bischof H, Holzapfel GA: A phenomenological approach toward patient-specific computational modeling of articular cartilage including collagen fiber tracking. ASME Journal of Biomechanical Engineering 2009, 131(9):091006.

47. Franks NR, Deneubourg JL: Self-organizing nest construction in ants: individual worker behaviour and the nest's dynamics. Animal Behaviour 1997, 54:779-796.

48. Deneubourg JL, Goss S, Franks N, Pasteels JM: The blind leading the blind: Modeling chemically mediated army ant raid patterns. Journal of Insect Behavior 2005, 2:719-725.

49. Reynolds CW: Flocks, Herds, and Schools: A Distributed Behavioral Model. Computer Graphics 1987, 21(4):25-34.

50. Partridge BL: The Structure and Function of Fish Schools. Scientific American 1982, 246(6):114-123.

51. Terborgh J: "Mixed flocks and polyspecific associations: Costs and benefits of mixed groups to birds and monkeys". American Journal of Primatology 2005, 21(2):87-10

52. Potts WK: The Chorus-Line Hypothesis of Manoeuvre Coordination in Avian Flocks. Letter in Nature 1984, 309:344-345.

53. Lukeman R, Yue-Xian L, Edelstein-Keshet L: Inferring individual rules from collective behaviors. Proceedings of the National Academy of Sciences 2010, 107:12576-12580.

54. Ballerini M, Cabibbo N, Candelier R, Cavagna A, Cisbani E, Giardina I, Lecomte V, Orlandi A, Parisi G, Procaccini A, Viale M, Zdravkovic V: Interaction ruling animal collective behavior depends ontopological rather than metric distance: Evidence from a field study. Proceedings of the National Academy of Sciences 2008, 105:1232-1237.

55. Helbing D, Farkas I, Vicsek T: Simulating dynamical features of escape panic. Nature 2000, 407:487-490.

doi:10.1186/1742-9994-8-3

Cite this article as: Kastberger et al:: Stereoscopic motion analysis in densely packed clusters: 3D analysis of the shimmering behaviour in Giant honey bees. Frontiers in Zoology 2011 8:3. 